
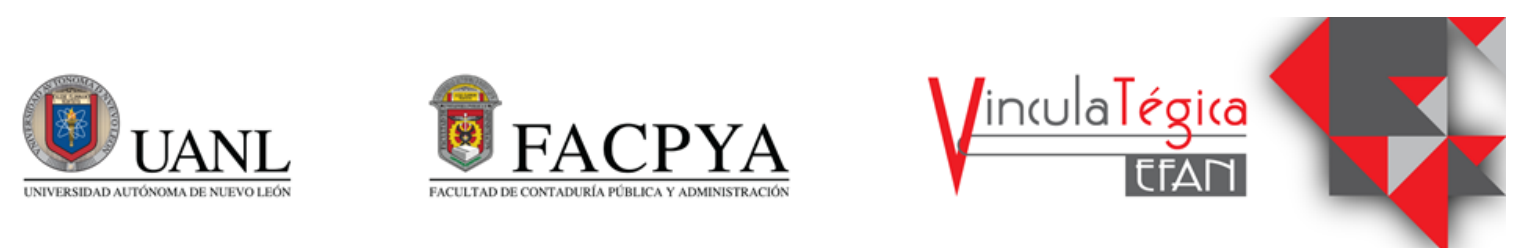

\title{
Determinantes de la adquisición de vivienda en el Área Metropolitana de Monterrey 2021
}

\author{
Sofía Alejandra Lugo Ortiz ${ }^{1}$ y Lizbeth Míreles Martínez ${ }^{2}$ \\ ${ }^{1}$ Facultad de Arquitectura, Universidad Autónoma de Nuevo León, sofia.lugoortz@uanl.edu.mx, Ciudad \\ Universitaria, Pedro de Alba s/n San Nicolás de los Garza, Nuevo León C.P. 66451, (81) 8329-4000 \\ ${ }^{2}$ Facultad de Arquitectura, Universidad Autónoma de Nuevo León, manaliz@yahoo.com, Ciudad \\ Universitaria, Pedro de Alba s/n San Nicolás de los Garza, Nuevo León C.P. 66451, (81) 8329-4000
}

Información del artículo revisado por pares

Fecha de aceptación: junio-2021

Fecha de publicación en línea: diciembre-2021

DOI: https://doi.org/10.29105/vtga7.1-125

\section{Resumen}

El objetivo de esta investigación es analizar el impacto que tiene la decisión de compra (demanda) de un inmueble (vivienda) en el área metropolitana de Monterrey ejerce sobre su valorización (sobrevalorización). La hipótesis es que la razón principal para la adquisición de vivienda en el AMM es el crecimiento personal a través de propiedades de mayor valor. Con base en la aplicación de la encuesta Factores para la determinación del precio de una vivienda en el AMM, se llevó a cabo un análisis estadístico inferencial que describen los principales factores que influyen en la decisión de adquisición de vivienda en el AMM. Esta información será de utilidad para el sector inmobiliario del área metropolitana, así como también para las personas interesadas en comprar o vender un bien inmueble. El documento se divide en secciones; la primera presenta la introducción, en seguida se revisa el marco teórico, el tercer apartado describe los materiales y métodos, la penúltima sección presenta los resultados y, finalmente, se concluye.

Palabras clave: área metropolitana, compra, vivienda.

\section{JEL: G1, G10, G12}

\begin{abstract}
The objective of this research is to analyze the impact that the decision to purchase (demand) a property (home) in the metropolitan area of Monterrey has on its appreciation (overvaluation). The hypothesis is that the main reason for home ownership in the AMM is personal growth through higher value properties. Based on the application of the survey Factors for determining the price of a home in the AMM, an inferential statistical analysis was carried out that describes the main factors that influence the decision to purchase a home in the AMM. This information will be useful for the real estate sector in the metropolitan area,
\end{abstract}


as well as for people interested in buying or selling real estate. The document is divided into sections; the first presents the introduction, then the theoretical framework is reviewed, the third section describes the materials and methods, the penultimate section presents the results and, finally, it concludes.

\section{INTRODUCCIÓN}

Actualmente el crecimiento habitacional ha tenido un gran impacto en el país, las construcciones de vivienda no paran y van a la par con el aumento de la población, el departamento de Asuntos Económicos y Sociales de la Organización de Naciones Unidas nos informa que la población urbana ha aumentado de forma exponencial y una de las áreas más urbanizadas a nivel internacional es América Latina y el Caribe con el $81 \%$ (ONU, 2018).

México ocupa el lugar número 11 en población a nivel mundial, por debajo de Japón y por encima de Etiopía, permaneciendo en el mismo lugar con respecto a 2010 (INEGI, 2020).

No es casualidad que los precios de vivienda vayan en aumento cuando es una necesidad, pero su sobrevaloración va más allá. La Sociedad Hipotecaria Federal (SHF) señala que en el 2020 se mostraron diferentes resultados en cada entidad federativa, donde 23 estados obtuvieron variaciones mayores a la nacional, teniendo en su cuarto trimestre una apreciación de $5.4 \%$ a nivel nacional diferente al año 2019 y como acumulado anual el 5.8\%
Keywords: metropolitan area, purchase, living place.

\section{JEL: GI, G10, G12}

a nivel nacional en el 2020. Mientras que, en el Área Metropolitana de Monterrey (AMM) el índice SHF de precios de la vivienda aumentó un $7.1 \%$, incrementando el precio del inmueble (SHF, 2021).

Analizando dicho incremento, en esta investigación remontamos a cuestionar ¿Qué factores impactan e influyen en la decisión de compra? Como hipótesis, se cree que uno de los factores primordiales para tomar tan importante decisión es el apego familiar, donde querer vivir cerca de un núcleo los hace sentir seguros y tranquilos, pero otro de los factores primordiales es el crecimiento personal, querer mejorar generación a generación y eso los lleva a adquirir propiedades de mayor valor.

En este estudio se busca encontrar respuestas claras y concisas de las variables a analizar con el objetivo idóneo de identificar, analizar y entender los determinantes de la adquisición de vivienda en el AMM. La investigación tomará fuerza mediante resultados obtenidos de una encuesta que estaremos aplicando a los habitantes del AMM, seguido dichos datos serán recabados para aplicar análisis estadístico descriptivo. 
Y tiene como objetivo analizar los componentes más importantes que la población del AMM toma al momento de decidir adquirir su vivienda.

\section{MARCO TEÓRICO}

La extensión de las ciudades mexicanas tuvo un incremento sin precedente en los últimos treinta años. En tres décadas, la superficie urbana creció a un ritmo de 50 hectáreas diarias, y si la población se duplicó en ese periodo, la superficie cubierta por la urbanización en México se multiplicó por diez (Sedesol, 2012).

El antecedente teórico sobre la valoración del precio de la vivienda se origina en el año 2007 se dio un auge de investigaciones sobre este tema. Escrivá (2009) señala que la economía mexicana, al igual que el resto del mundo, está pasando por un periodo de recesión, el cual ha tenido un impacto negativo en algunos de los principales sectores que componen la actividad económica del país. Sin embargo, el sector inmobiliario mexicano no se vio fuertemente afectado por la situación económica global del año 2008.

Esta afirmación es fundamentada por el hecho de que las ventas de casas se ubicaron muy cerca de los máximos históricos. Aun así, de acuerdo con Escrivá (2009), la volatilidad de los precios se ha visto influenciada por la incertidumbre, tanto de inversionistas, como de los propios consumidores.
Después de la crisis de 1995 en donde se registró una caída superior al 20\%. Aun así, Nuevo León representa el segundo lugar nacional en aportación al producto interno bruto (PIB) en ese ramo. Similarmente, en cuanto al total de empleos generados en esta industria, este estado también se coloca en segundo lugar nacional, solamente antecedido por el Distrito Federal. Debido al desempeño del estado en este sector de la economía, resulta relevante estudiar el impacto de la crisis actual, en específico para el área metropolitana de Monterrey, ya que ahí se encuentra concentrado más del $90 \%$ del valor de la construcción residencial en esta entidad de Nuevo León (Favela,2010 y Escrivá, 2009).

Rosen (1974) y Moreno y Alvarado (2011) señala que la función de precios hedónicos se define como el precio de mercado, el cual varía en función a las diferentes características que éste posee, es decir, la demanda de atributos determina el cambio en los precios.

Kain y Quigley (1975) identifican cuatro componentes principales que determinan el precio de una vivienda. Estos son: estructura, calidad, tamaño y características del vecindario. 
El pensamiento social viene de la interacción entre los individuos y funda los modos de funcionamiento de una sociedad en la vida cotidiana, que es compartida por la construcción intersubjetiva que se da, precisamente, como producto de las interacciones y la comunicación de las personas (Chávez, 2007 y Araya, 2002).

\section{MATERIAL Y MÉTODOS}

Para alcanzar el objetivo propuesto en esta investigación y determinar el efecto de la percepción de los ciudadanos del AMM se creó una encuesta que nos hace cuestionar ¿Cómo influye la sobrevaloración del precio de la vivienda en el AMM? Para poder dar respuesta a esta pregunta nos dimos a la tarea de aplicarla a los ciudadanos del AMM para así conocer las variables que impactan en la sobrevaloración del precio de una vivienda en la actualidad.

Dicha investigación tiene una base de datos de 199 encuestados y así mismo, la encuesta que está diseñada bajo un esquema de muestreo no probabilístico por conveniencia el cual nos permite analizar los factores demográficos, localización y variables de estudio, como: intereses personales.

Posteriormente, se aplica un estadístico descriptivo de frecuencias con el objetivo de cuantificar los efectos de las variables explicativas sobre la sobrevaloración del precio de la vivienda en el AMM.

\section{Tabla 1. Descripción de variables}

\begin{tabular}{ll}
\hline \multicolumn{1}{c}{ Variable } & \multicolumn{1}{c}{ Definición } \\
\hline Género & $\begin{array}{l}\text { Término técnico específico en ciencias sociales que alude al } \\
\text { conjunto de características diferenciadas que cada sociedad asigna } \\
\text { a hombres y mujeres. }\end{array}$ \\
Estado civil & $\begin{array}{l}\text { Situación de las personas físicas determinada por sus relaciones } \\
\text { de familia, provenientes del matrimonio o del parentesco, que } \\
\text { establece ciertos derechos y deberes. }\end{array}$ \\
Edad & $\begin{array}{l}\text { Tiempo que ha vivido una persona u otro ser vivo contando desde } \\
\text { su nacimiento. }\end{array}$ \\
Escolaridad & $\begin{array}{l}\text { Período de tiempo que un niño o un joven asiste a la escuela para } \\
\text { estudiar y aprender, especialmente el tiempo que dura la } \\
\text { enseñanza obligatoria. }\end{array}$ \\
Ingreso mensual & $\begin{array}{l}\text { Se refiere al conjunto de ingresos netos percibidos por todos los } \\
\text { miembros de la familia, aporten o no estos ingresos o parte de los } \\
\text { mismos para sufragar los gastos. }\end{array}$ \\
Situación laboral & $\begin{array}{l}\text { Situación en la que se encuentran las personas en edad laboral en } \\
\text { relación con el mercado de trabajo. }\end{array}$ \\
Vive & Lugar actual donde habita una persona.
\end{tabular}


Le gustaría vivir

Vínculo de apego

Segmento

Factores

Importancia de ubicación para la convivencia social

Para vivir (vivienda principal)

Segunda residencia
Lugar donde le gustaría vivir a una persona en corto, mediano o largo plazo.

La cercanía con la otra persona brinda resguardo y seguridad.

Hace referencia al sector habitacional donde prefiere vivir una persona.

Importancia de accesibilidad y/o cercanía que considera la población al momento de elegir su vivienda ideal.

La sociedad considera importante el lugar donde vive para mejor sus relaciones sociales.

El objetivo de compra es como vivienda principal, para formar una familia o independizarse.

Buscan tener otro patrimonio para cambiarse a futuro y vender la actual.

Fuente: Elaboración propia.

\section{RESULTADOS}

\subsection{Estadísticos}

De acuerdo con el análisis descriptivo de las variables antes mencionadas podemos observar que el $68.8 \%$ de los encuestados su grado de escolaridad es de Licenciatura y el
$59.8 \%$ son de género femenino donde el estado civil predominante es soltero(a) con un $55.8 \%$, esto conlleva que el $55.8 \%$ de los agentes tienen un empleo de tiempo completo ganando alrededor de $\$ 15,000$ a $\$ 25,000$ pesos según tabla 2.

Tabla 2. Estadísticos descriptivos de las variables

\begin{tabular}{llll}
\hline Variable & Rango & Frecuencia & Porcentaje \\
\hline Género & Hombre & 80 & $40.2 \%$ \\
& Mujer & 119 & $59.8 \%$ \\
& Total & 199 & $100 \%$ \\
Estado civil & & & \\
& Soltero(a) & 111 & $55.8 \%$ \\
& Casado(a) & 79 & $39.7 \%$ \\
& Divorciado(a) & 8 & $4.0 \%$ \\
& Viudo(a) & 1 & $0.5 \%$ \\
& Total & 199 & $100 \%$
\end{tabular}




\begin{tabular}{|c|c|c|c|}
\hline \multirow[t]{6}{*}{ Edad } & 16 a 26 años & 67 & $33.7 \%$ \\
\hline & 27 a 36 años & 80 & $40.2 \%$ \\
\hline & 37 a 46 años & 33 & $16.6 \%$ \\
\hline & 47 a 58 años & 17 & $8.5 \%$ \\
\hline & Más de 58 años & 2 & $1.0 \%$ \\
\hline & Total & 199 & $100 \%$ \\
\hline \multirow[t]{6}{*}{ Escolaridad } & Primaria & 2 & $1.0 \%$ \\
\hline & Secundaria & 3 & $1.5 \%$ \\
\hline & Preparatoria & 9 & $4.5 \%$ \\
\hline & Licenciatura & 137 & $68.8 \%$ \\
\hline & Posgrado & 48 & $24.1 \%$ \\
\hline & Total & 199 & $100 \%$ \\
\hline \multirow{7}{*}{ Ingreso mensual } & Menos de $\$ 5,000$ pesos & 20 & $10.1 \%$ \\
\hline & $\$ 5,000$ a $\$ 15,000$ pesos & 55 & $27.6 \%$ \\
\hline & $\$ 15,000$ a $\$ 25,000$ pesos & 64 & $32.2 \%$ \\
\hline & $\$ 25,000$ a $\$ 35,000$ pesos & 19 & $9.5 \%$ \\
\hline & $\$ 35,000$ a $\$ 40,000$ pesos & 14 & $7.0 \%$ \\
\hline & Más de $\$ 40,000$ pesos & 20 & $10.1 \%$ \\
\hline & Total & 192 & $96.5 \%$ \\
\hline \multirow[t]{7}{*}{ Situación laboral } & Estudiante & 17 & $8.5 \%$ \\
\hline & Empleo de medio tiempo & 5 & $2.5 \%$ \\
\hline & Empleo de tiempo completo & 111 & $55.8 \%$ \\
\hline & Trabajador por cuenta propia & 58 & $29.1 \%$ \\
\hline & Desempleado & 7 & $3.5 \%$ \\
\hline & Jubilado & 1 & $0.5 \%$ \\
\hline & Total & 199 & $100 \%$ \\
\hline
\end{tabular}

Fuente: Elaboración propia.

\subsection{Localización}

Uno de los principales factores al momento de elegir lugar donde vivir es la localización; es por ello que en la encuesta aplicada se hace un comparativo de percepción del lugar donde se habita actualmente y donde les gustaría habitar a corto, mediano o largo plazo ver Gráfica 1. 
Gráfica 1. Comparativo de percepción del lugar donde se habita y donde les gustaría habitar

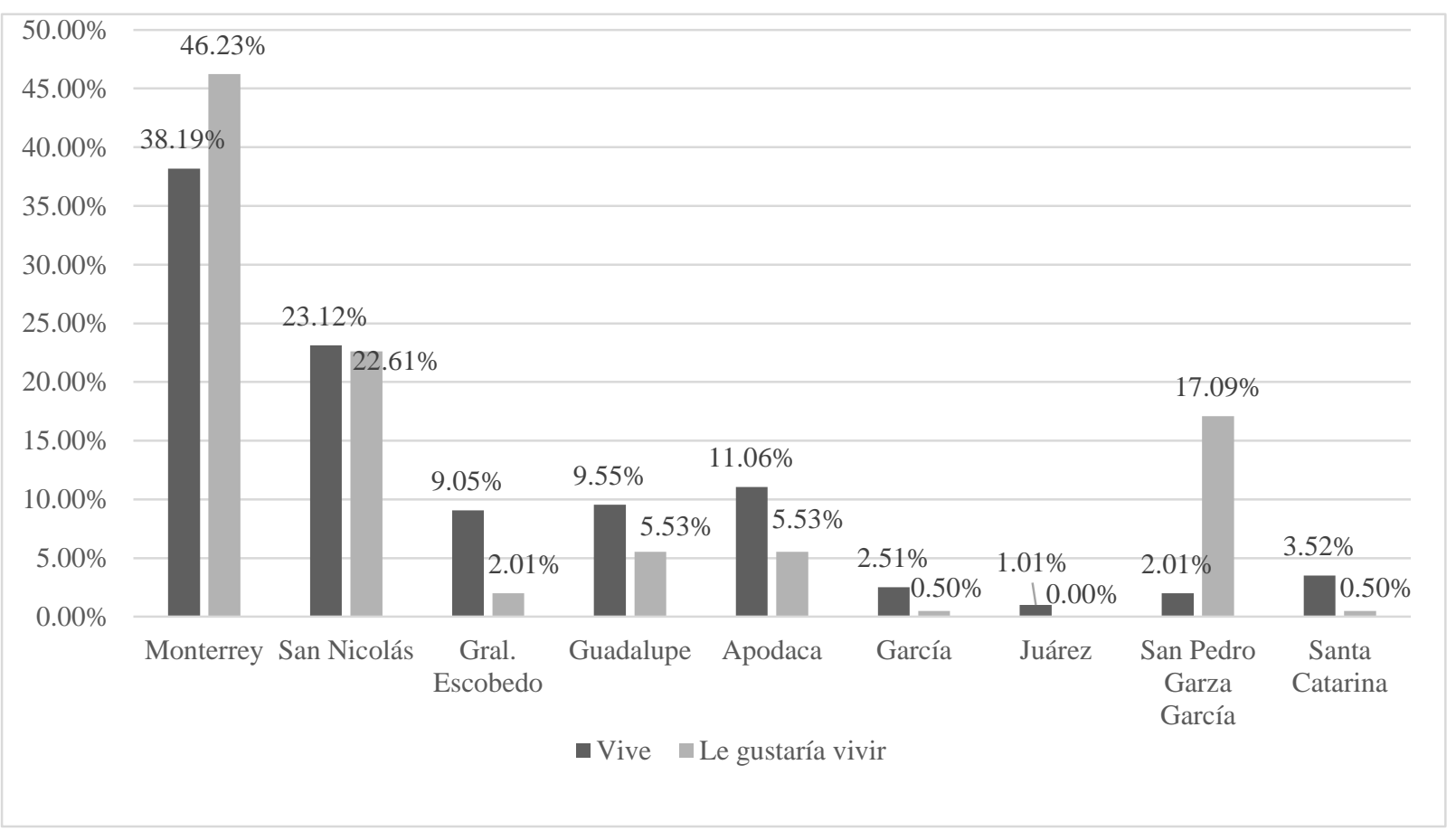

Fuente: Elaboración propia

En base a los resultados de la Grafica 1, podemos observar que el $38 \%$ vive en el municipio de Monterrey y asimismo el $46.2 \%$ le gustaría cambiarse o adquirir una segunda vivienda en el mismo municipio, esto nos indica que la mayoría de los encuestados prefiere quedarse cerca.

Como cultura del área metropolitana tendemos a tener ciertos apegos que son de total influencia al momento de elegir vivienda, ver la siguiente tabla.

\begin{tabular}{llll}
\hline Variable & Rango & Frecuencia & Porcentaje \\
\hline Vínculo de apego & Familiares & 103 & $51.8 \%$ \\
& Lugar de trabajo & 79 & $39.7 \%$ \\
& Amigos & 17 & $8.5 \%$ \\
Total & 199 & $100 \%$ \\
Segmento & Fraccionamiento privado & 124 & $62.3 \%$ \\
& Colonia tradicional & 68 & $34.2 \%$ \\
Tactores & Total & 192 & $96.5 \%$ \\
& & & \\
& Rápido acceso a vías principales & 159 & $79.9 \%$ \\
& Cercanía a tienda de conveniencia & 28 & $14.1 \%$ \\
& Cercanía a clínicas/hospitales & 5 & $2.5 \%$ \\
& Cercanía a escuelas & 7 & $3.5 \%$ \\
& Total & 199 & $100 \%$
\end{tabular}




\begin{tabular}{llll} 
Importancia de ubicación para & Sí & 183 & $92 \%$ \\
la convivencia social & No & 15 & $7.5 \%$ \\
& Total & 198 & $99.5 \%$ \\
\hline
\end{tabular}

Fuente: Elaboración propia

Tabla 2. Característica social de ubicación

De acuerdo con la Tabla 2, es posible afirmar que los habitantes del AMM poseen el comportamiento de mantenerse cerca del seno familiar cuando se decide adquirir una vivienda, puesto que el $52 \%$ considera esta condición al momento de elegir su vivienda. Asimismo, la cercanía con el trabajo como mecanismos para reducir tiempos de tráfico, ahorro tanto de gasolina o transporte público, reporto el $39.7 \%$ de las preferencias en cuanto a motivaciones para adquirir vivienda se refiere.

\subsection{Datos específicos}

Para la decisión de compra debemos analizar cuáles son las principales razones por lo cual
En cuanto al segmento, la mayoría de los encuestados del AMM (62\%) indicaron que prefieren vivir en un fraccionamiento privado.

Además, el $80 \%$ de los encuestados destacó que el acceso a vías principales es el principal factor que consideran a la hora de adquirir una vivienda y el $92 \%$ da importancia a la ubicación de su vivienda para atender temas de convivencia social.

Gráfica 2. Motivo de compra.

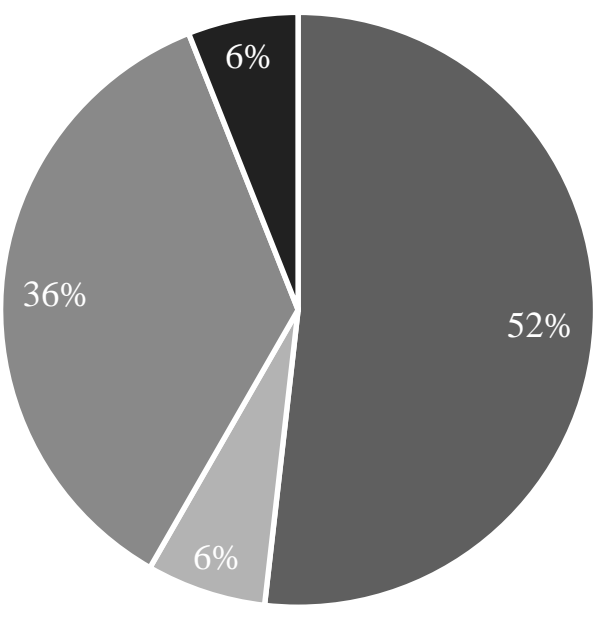

- Para vivir (vivienda principal) - Segunda residencia

- Como inversión/para alquilar - Para transmitir a mis hijos 
Fuente: Elaboración propia.

La Gráfica 2 señala los motivos de compra para adquirir una vivienda y destaca la motivación de vivienda principal como la motivación esencial de los habitantes del AMM con un $52 \%$, lo que podría asociarse a decisiones de esa vivienda principal, es decir que la población está creciendo, buscando independizarse $y / o$ formando familia.
No obstante, la influencia y razones que tiene la población a la hora de hacer su compra juegan un papel muy importante y determinante, es por eso que en la siguiente grafica veremos las principales razones por las que se decide hacer la compra de manera concisa, ver Grafica 3.

Gráfica 3. Influencia de compra

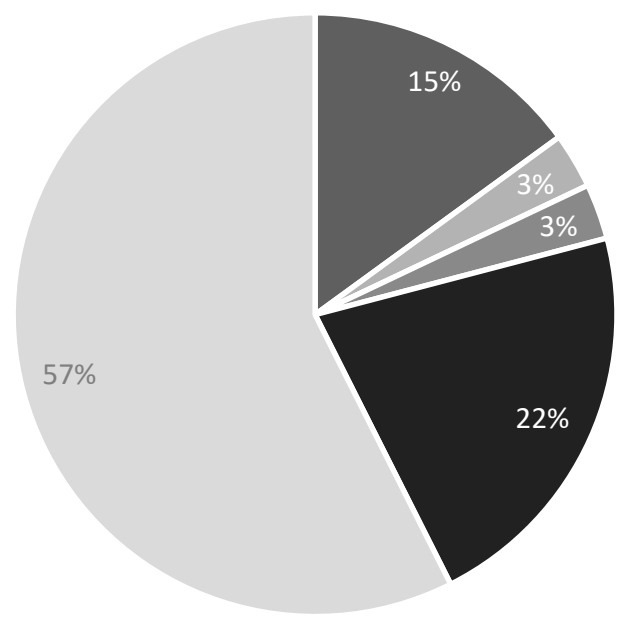

- En el futuro no podría comprar

- Conocidos habían comprado recientemente

• En un futuro serán más caras
- Conversaciones con amigos

- Noticias sobre el precio de la vivienda

Fuente: Elaboración propia.

La influencia de la decisión inmediata que vemos en la Gráfica 3 al comprar una vivienda, la mayoría de los encuestados (57\%) opta por no pensarlo mucho porque en un futuro serán más costosas y el segundo porcentaje más alto (22\%) realiza su compra inmediata por noticias sobre el precio actual de la vivienda, ambos resultados están relacionados con tema económico. 


\section{Gráfica 4. Riesgo de compra}

Fuente: Elaboración propia.

Después de analizar la Grafica 3 vemos como la población puede sentir algún tipo de riesgo en su economía al momento de comprar su vivienda. En la Gráfica 4 observamos que el riesgo moderado-normal que la población ve al adquirir una vivienda es del $54 \%$ lo cual puede deberse a la situación económica tal como vimos con anterioridad en la Gráfica 3.

\section{CONCLUSIONES}

Con el análisis de dicho estudio, podemos observar las variables analizadas para entender los determinantes que existen a la hora de decidir adquirir una vivienda en el área metropolitana de Monterrey.

De acuerdo a los factores que tuvieron mayor influencia al momento de tomar una decisión de compra que nos arrojó nuestro método estadístico descriptivo por medio de una encuesta a 199 personas, comprobamos nuestra hipótesis de que la mayor influencia al posen el comportamiento de mantenerse cerca del seno familiar como lo mencionamos con anterioridad, así como querer mejorar generación tras generación llevándonos a que hoy en día en el AMM las nuevas generaciones quieren y prefieren vivir en fraccionamientos privados.

La información obtenida puede ser utilizada para inversionistas del AMM para plantear mejores estrategias en sus planes de fraccionamientos de vivienda o bien para que

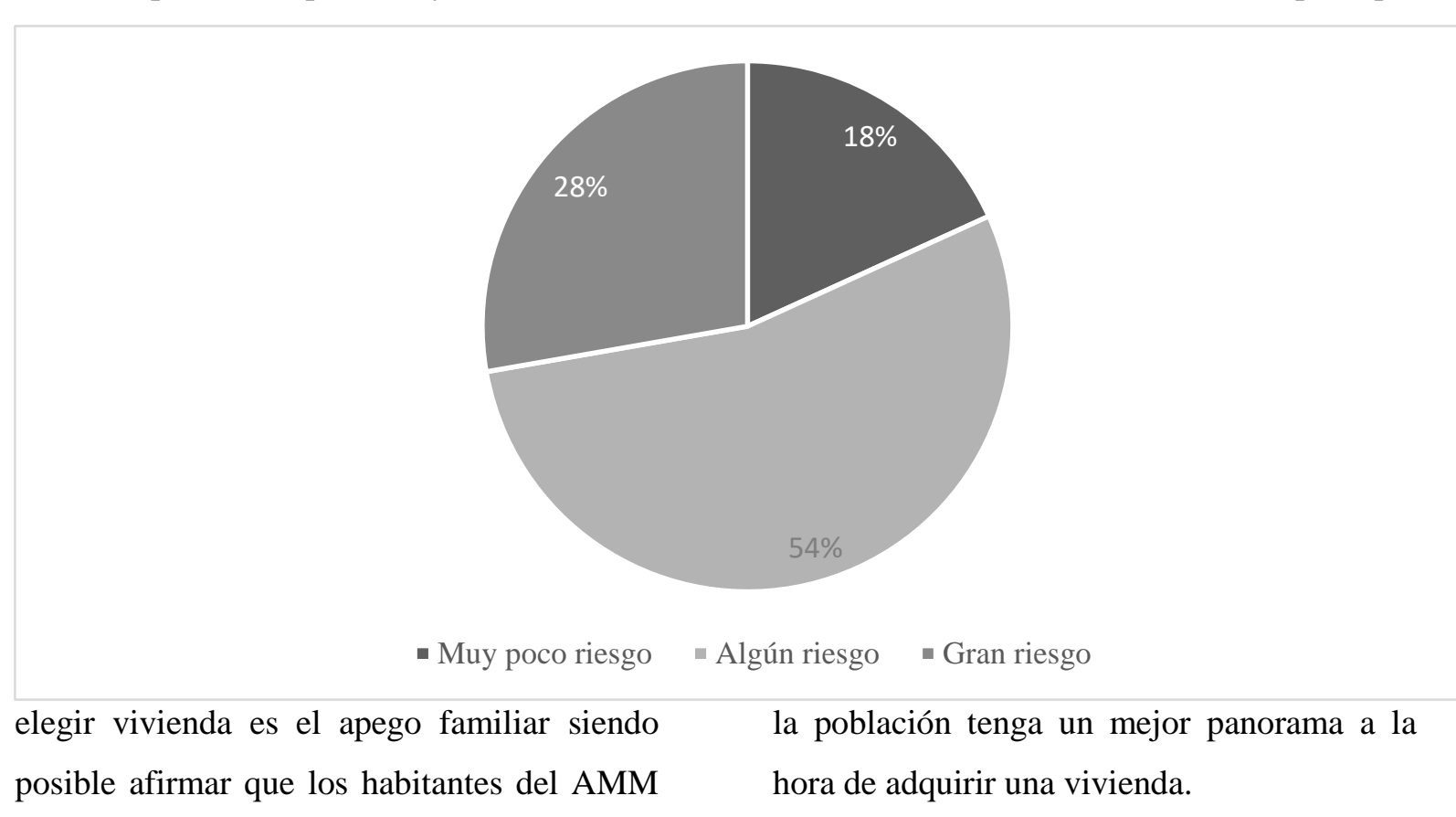




\section{REFERENCIAS}

Araya, S. (2002). Las representaciones sociales: Ejes teóricos para su discusión. Sede Académica, Costa Rica Sitio web: http://www.efamiliarycomunitaria.fcm.unc.edu.ar/libros/Araya\%20Uma\%F1a\%20Representa ciones\%20sociales.pdf

Chávez, A y Ortega, R. (2007). Representaciones sociales de estilos de vida y bienestar en el contexto de una sociedad de consumo: Monterrey y su área metropolitana, Sitio web: http://www.scielo.org.mx/pdf/crs/v13n25/2007-8110-crs-13-25-76.pdf

Escrivá (2009). Situación Inmobiliaria. Servicio de Estudios Económicos. Octubre de 2009. Recuperado el 3 de septiembre de 2009, de la base de datos BBV A Bancomer. Sitio web: https://www.bbvaresearch.com/wpcontent/uploads/mult/0910_SituacionInmobiliariaMexico_ 16_tcm346-203326.pdf

Instituto Nacional de Estadística y Geografía (INEGI). (2020). Censo de Población y Vivienda 2020 Sitio web: https://www.inegi.org.mx/programas/ccpv/2020/\#Documentacion

Kain, J.F. y Quigley, J.M. (1975) Housing Markets and Racial Discrimination: A Microeconomic Analysis. Recuperado el 6 de octubre de 2009, de la base de datos NBER Sitio web: http://www.nber.org/books/kain75-1

Moreno, R. y Alvarado, E. (2011). El entorno social y su impacto en el precio de la vivienda: un análisis de precios hedónicos en el área metropolitana de Monterrey. Trayectorias, (14)33, 131-147.

Organización de las Naciones Unidas (ONU). (2018). Las ciudades seguirán creciendo, sobre todo en los países en desarrollo. Sitio web: https://www.un.org/development/desa/es/news/population/2018-world-urbanizationprospects.html

Rosen, S. (1974). Hedonic Prices and Implicit Markets: Product Differentiation in Puré Competition. The Journal of Political Economy, 82 (1): 34-55. Recuperado el 6 de octubre de 2009, de la base de datos IDEAS. Sitio web: http://neconomides.stern.nyu.edu/networks/phdcourse/Rosen_Hedonic_prices.pdf

Sedesol (2012), La expansión de las ciudades 1980-2010, México, Secretaría de Desarrollo Social. Sitio

web: https://www.conavi.gob.mx/images/documentos/transparencia/rendicion cuentas/DECRETO \%20por\%20el\%20que\%20se\%20aprueba\%20el\%20Programa\%20Sectorial\%20de\%20Desar rollo\%20Agrari.pdf

Sociedad Hipotecaria Federal (SHF) (2021). Índice SHF de precios de la vivienda en México, cuarto trimestre de 2020., Sitio web: https://www.gob.mx/shf/articulos/indice-shf-de-precios-de-lavivienda-en-mexico-cuarto-trimestre-de-2020-263700?idiom=es 